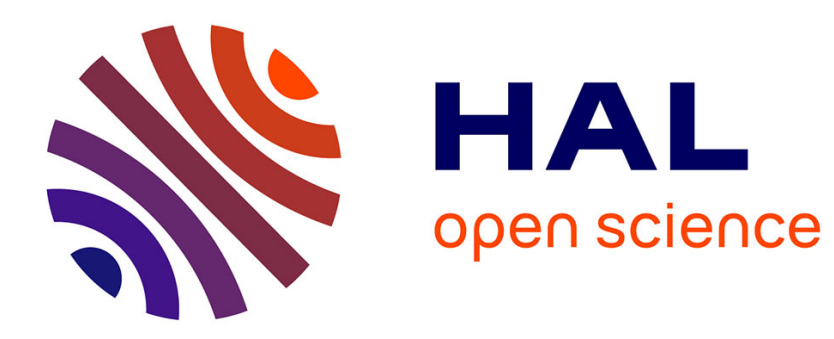

\title{
Inégalités sociales et vie augmentée
}

Bernard Lahire

\section{To cite this version:}

Bernard Lahire. Inégalités sociales et vie augmentée. Revue française des affaires sociales, 2021, Juillet-Septembre 2021. hal-03339939

\section{HAL Id: hal-03339939 \\ https://hal.science/hal-03339939}

Submitted on 28 Oct 2021

HAL is a multi-disciplinary open access archive for the deposit and dissemination of scientific research documents, whether they are published or not. The documents may come from teaching and research institutions in France or abroad, or from public or private research centers.
L'archive ouverte pluridisciplinaire HAL, est destinée au dépôt et à la diffusion de documents scientifiques de niveau recherche, publiés ou non, émanant des établissements d'enseignement et de recherche français ou étrangers, des laboratoires publics ou privés. 
[POINT DE VUE]

Inégalités sociales et vie augmentée

Bernard Lahire

Une fausse citation attribuée à Steve Jobs, cofondateur d'Apple mort le 5 octobre 2011, est régulièrement partagée sur les réseaux sociaux depuis quelques années ${ }^{1}$. Censé avoir été prononcé au cours des dernières heures de sa vie, ce monument de démagogie se présente comme un grand moment de sagesse : un appel à apprécier la vie plutôt que la richesse ; une sorte de développement du thème proverbial : « L'argent ne fait pas le bonheur. » L'une des versions (abrégée) que l'on trouve sur les réseaux sociaux est la suivante :

« J'ai atteint le summum du succès dans le monde des affaires. Dans les yeux des autres, ma vie est une réussite. Cependant, mis à part le travail, j'ai eu peu de joie. En fin de compte, la richesse n'est qu'un fait auquel je me suis habitué. En ce moment, allongé sur mon lit d'hôpital, et me rappelant toute ma vie, je me rends compte que toute la reconnaissance et la richesse dans laquelle j'ai pris tant de fierté, a pâli et est devenue insignifiante face à la mort imminente. Vous pouvez employer quelqu'un pour conduire votre voiture ou gagner de l'argent pour vous mais c'est impossible d'engager quelqu'un pour supporter la maladie et mourir pour vous. Les choses matérielles perdues peuvent être retrouvées. Mais il y a une chose qui ne peut jamais être trouvée quand elle est perdue : "la vie". Quelle que soit l'étape de la vie à laquelle nous sommes actuellement, avec le temps, nous serons confrontés au jour où le rideau se ferme. Aimez votre famille, votre conjoint et vos amis... Traitez-les bien. Chérissez-les. Au fur et à mesure que nous vieillissons, et devenons plus sages, nous réalisons lentement que porter une montre à $300 \$$ ou $30 \$$ ne change rien : les deux donnent la même heure... Que nous ayons un portefeuille ou un sac à main de $300 \$$ ou de 30 \$, son contenu est le même. Que nous conduisions une voiture de $150000 \$$ ou une voiture de $30000 \$$, la route et la distance sont les mêmes, et nous arrivons à la même destination. Que nous buvions une bouteille de vin à $1000 \$$ ou à $10 \$$, la gueule de bois est la même. Que la maison dans laquelle nous vivons mesure 300 ou 3000 pieds carrés, la solitude est la même. Vous réaliserez que votre véritable bonheur intérieur ne provient pas des choses matérielles de ce monde. Que vous voyagiez en première classe ou en classe économique, si l'avion tombe en panne, vous tombez avec lui... [...] N'éduquez pas vos enfants

1. Les Décodeurs, « Cette citation que Steve Jobs n’a jamais prononcée sur son lit de mort », Le Monde, 31 juillet 2019. 
à être riches. Éduquez-les à être heureux. Ainsi, quand ils grandiront, ils connaîtront la valeur des choses et non leur prix. »

La rhétorique de ce texte consiste à rappeler une évidence (riche ou pauvre, tout le monde meurt) et à effacer les effets réels produits par les inégalités économiques sur la santé, l'espérance de vie et, plus précisément, l'espérance de vie en bonne santé. La fonction d'un tel discours est de déconnecter totalement la question de « la vie » (de sa durée, de son confort, des moyens de diminuer les souffrances physiques et psychiques, etc.), de celle des inégalités sociales, et notamment économiques. En s'appuyant sur des exemples qui renvoient la richesse à une pure stratégie de distinction ou d'esbroufe sociale, sans conséquences réelles (À quoi sert de porter une montre très chère si l'heure peut être donnée par une montre à bas prix ? À quoi sert de porter un sac très chic quand un sac très ordinaire remplit la même fonction ? À quoi sert de vivre dans une grande maison si l'on connaît la solitude ?) ; en masquant le fait que les voitures ne sont pas des moyens de transports abstraits mais des habitats plus ou moins agréables, spacieux, pouvant, dans certains cas, diminuer les douleurs lombaires, de même que l'espace restreint ou vaste des maisons ou des places d'avion « en première classe » ou en « classe économique » ne sont pas réductibles à des signes de richesse purement ostentatoires, mais qu'ils ont des conséquences, au jour le jour et à long terme, sur les corps de ceux qui vivent dans ces espaces ; et, last but not least, en se gardant bien d'évoquer tous les effets directs sur la santé et sur la vie (sa durée et sa qualité) de tous les soins chirurgicaux, médicaux, paramédicaux, etc., auxquels permet d'accéder la richesse d'une nation ou d'un individu, la prétendue « sagesse » de Steve Jobs, au seuil de la mort, contribue à déréaliser les effets des inégalités et à occulter les avantages tout à fait réels sur « la vie » associés à la richesse économique. L'argent - cet « équivalent universel » dont parlait Marx - est, dans les sociétés capitalistes, le capital des capitaux et, en tant que tel, ce qui permet d'accéder à toutes les formes possibles de ressources, des plus matérielles aux plus culturelles, en passant par les divers services domestiques, éducatifs, médicaux, techniques, etc. En posséder beaucoup donne un pouvoir déterminant sur la vie.

L'exemple de ce faux « morceau de sagesse » contemporaine, qui peut paraître caricatural de démagogie, est cependant loin d'être déconnecté des problèmes que l'on rencontre dans l'analyse sociologique des inégalités sociales de toute nature. En effet, certaines analyses pèchent par leur " artificialisme » et une certaine forme de déréalisation de leur objet. Parmi l'ensemble des inégalités sociales, celles touchant à la santé, qui concernent des questions vitales, apparaissent particulièrement utiles pour prendre conscience de telles dérives interprétatives.

\section{Inégalités et différences avantageuses}

Que l'on se tourne vers la paléoanthropologie, la préhistoire, l'ethnologie, l'histoire ou la sociologie, force est de constater que pas une seule société humaine 
connue n'a échappé aux inégalités qu'elle qu'en soit la nature, et l'idée selon laquelle il pourrait y avoir eu, dans le passé, un âge d’or des sociétés sans inégalité ni domination a été balayée par les faits. Ceux qui parlent de sociétés humaines égalitaires, comme le fait par exemple l'ethnoarchéologue Brian Hayden, ne parlent en définitive que des inégalités de type économique (Hayden, 2013), en oubliant que les inégalités entre les sexes, entre les classes d’âge, entre experts et profanes, entre groupes ethniques, familles ou lignages, etc., n’ont cessé de tramer les sociétés, des plus anciennes aux plus récentes. Que ces formes d'inégalités varient historiquement est une évidence. Qu'elles aient pris une dimension économique particulièrement marquée depuis la révolution néolithique (il y a environ 10000 ans), avec la sédentarité, l'agriculture et l'élevage, qui permettent la domestication de la nature et la constitution de stocks alimentaires, ainsi que la densification démographique qui s'accompagne d'une plus grande division du travail (Testart, 2012 ; Demoule, 2017), est tout aussi certain. Mais les rapports de domination ne se limitent pas, loin de là, à des rapports économiques d'exploitation, ni même à des rapports politiques de domination qui ont pris une place importante depuis que des structures verticales fortement hiérarchisées ont vu le jour avec la formation des premiers États.

Quand elle fait correctement son travail, la sociologie met donc inévitablement au jour la réalité des dissymétries, des inégalités, des rapports de domination et d'exploitation, et les processus par lesquels ils parviennent à se reproduire ou à se transformer pour mieux se perpétuer. La question des inégalités n’est pas une question parmi d'autres que pourraient décider de traiter ou d'ignorer les sociologues. Elles ne sont pas de simples vues de l'esprit et une sociologie parfaitement irénique, ou ne voyant jamais ni dominations ni inégalités, ne serait tout simplement pas sociologie.

Certes, toute différence sociale constatée n'est pas interprétable en termes d'inégalité sociale. Pour qu'une différence sociale puisse faire inégalité, elle doit concerner des pratiques, des biens ou des ressources avantageuses. Ainsi, avoir les yeux bleu ou marron constitue une différence qui ne produit pas, en l'état actuel des choses, une situation qu'on peut qualifier d'inégalitaire. Pour qu'une différence devienne inégalité, il faut que la privation de telle caractéristique, de telle ressource matérielle, de tel bien culturel, de tel savoir ou de tel service constitue un désavantage, un manque ou un handicap.

Personne n'oserait affirmer que vivre plus longtemps, dans de meilleures conditions matérielles et sanitaires, avec des ressources économiques et culturelles suffisantes pour sa vie ou sa survie, n'est pas enviable. Et la question de savoir si celles et ceux qui sont victimes de ces privations les vivent avec un sentiment d'injustice et de révolte, ou avec fatalisme et soumission, est une tout autre question. Les perdants et les perdantes d'une structure sociale inégalitaire peuvent tout à fait penser, par intériorisation de leur état comme évidence, que leur situation est le fruit du hasard, de la fatalité, de la nature des choses ou de leur incapacité personnelle, alors même qu'elle tient à des mécanismes structurels et historiquement déterminés de production d’inégalités indépendantes de leur personne. 
Un certain « artificialisme », que dénonçait Jean-Claude Chamboredon, aurait toutefois tendance à faire des questions d'inégalités une pure affaire de convention. Même s'il est parfois utile de rappeler le caractère culturellement arbitraire de certaines conventions, de certains goûts ou de certaines habitudes, les dominants ont néanmoins toujours su s'approprier ce qu'il y avait de mieux à leur époque en termes de conditions de vie, de confort, de protection, d'alimentation, de soins, de sécurité, de bien-être, etc. Les inégalités tournent souvent autour de pouvoirs et de gains tout à fait réels du point de vue de la qualité des conditions même de la vie humaine (Lahire, 2019). Réduire les inégalités à de simples effets de classements ou à l'instauration d'une hiérarchie purement arbitraire des valeurs et des légitimités, ce serait totalement déréaliser la situation vécue par les dominants et les dominés.

Le monde ne se réduit pas à un grand jeu de classement à partir d'une hiérarchie des légitimités ou des valeurs. Jean-Claude Chamboredon rappelait pertinemment à ceux qui ne pensent tous les écarts qu'en termes de degré de légitimité que les différences entre classes sociales sont, certes, des jugements de valeur, mais toujours « inscrits dans des choses bien réelles » (Chamboredon, 1996). Par exemple, que ce soit dans le « morceau de sagesse » commenté dans l'introduction ou dans la « sociologie des légitimités ou des valeurs » (Bourdieu, 1979), les différences entre les voitures sont ramenées à des écarts de légitimité, pour dénoncer la futilité de ces différences dans le premier cas, et pour pointer les hiérarchies sociales traduites et légitimées en différences symboliques dans le second cas. Si la «vieille voiture Renault 4L et la Renault 25 ou la Rolls-Royce » peuvent être classées du moins chic au plus chic, ou du moins légitime au plus légitime, les différences entre elles ne sont pas seulement de l'ordre du degré de légitimité, mais concernent des vitesses, des conditions de sécurité et des conforts très inégaux. L'« artificialisme social » critiqué par Chamboredon n'est autre qu'un conventionnalisme excessif qui réduit toute réalité à sa valeur relative ou à son degré de légitimité en déréalisant les effets très pratiques de biens matériels ou symboliques sur les conditions de vie quotidiennes ; biens qui sont, par ailleurs, inégalement classés et classants.

\section{L'inégale distribution des formes d'augmentation de soi}

Que signifient au fond, anthropologiquement, ces inégalités et les processus de leur reproduction ? L'humanité, entendue comme espèce animale issue d'une très longue histoire évolutive, se distingue des autres espèces animales par sa capacité, non unique mais inégalée, à produire des artefacts de natures très diverses (outils, machines, armes, vêtements, habitats, etc.) ainsi que des dispositions, des savoirs et savoir-faire qui se transmettent d'une génération à l'autre et rendent possible un véritable processus d'accumulation culturelle à l'état objectivé, comme à l'état incorporé. Non seulement Homo sapiens accumule des artefacts et constitue un 
patrimoine de dispositions et de compétences incorporées, mais il les transmet aux nouvelles générations qui ne partent jamais de zéro. Comme disait Marx : « La tradition de toutes les générations mortes pèse d'un poids très lourd sur le cerveau des vivants » (Marx, 2017).

Or, que représentent tous ces savoirs et tous ces artefacts fabriqués par les hommes au cours d'une longue histoire cumulative ? Entourés par cette profusion de dispositifs objectivés et porteurs de multiples dispositions et compétences incorporées, nous finissons par ne plus voir ce que la culture cumulative nous fait. Et pourtant nous en sommes intimement dépendants, pour le meilleur - notre santé et l'allongement de notre durée de vie, notre capacité à décupler nos forces et nos sens, notre confort matériel, notre connaissance du monde, nos plaisirs, etc. - comme pour le pire - la dépendance aliénante à l'égard de tous les artefacts et de toutes les connaissances scientifiques, techniques ou bureaucratiques que nous ne maîtrisons pas, de même que les effets indésirables en retour de la profusion des artefacts sur notre vie et notre santé (asthme, perturbations endocriniennes, cancers, etc.).

Le mouvement « transhumaniste » qui prône le développement des techniques (mécaniques, informatiques, robotiques, etc.) permettant d'augmenter les capacités physiques et mentales humaines est le plus souvent associé à la question de l'avenir de l'humanité. Pourtant, c'est bien l'humanité moderne même (homo sapiens) qui, depuis près de 300000 ans, est indissociable d'artefacts tels qu'outils, armes, vêtements, habitats, techniques et savoirs (de chasse, de pêche, de fabrication ou de préservation du feu, etc.) permettant l'extension ou l'augmentation de soi, de ses capacités cognitives et de ses forces. Aller plus loin, plus haut, plus vite, par la domestication du cheval, par l'usage des chaussures, de la roue, de la voiture, de l'avion, de la fusée, etc. ; mieux voir et mieux entendre grâce à la médecine, aux lunettes, aux jumelles et aux prothèses auditives ; moins souffrir de maladies grâce encore à la médecine, à la chirurgie et aux médicaments ; communiquer quand la voix naturelle ne suffit plus par l'écrit, le télégraphe, le téléphone ou le courriel ; creuser la terre avec des outils malgré l'absence de griffes ; vivre dans les régions les plus froides grâce à des vêtements ou des techniques de chauffage malgré l'absence de peaux épaisses et de toisons protectrices ; déplacer des poids lourds avec des machines en dépit de sa faiblesse musculaire, etc. : voilà ce que l'humanité n'a cessé de rendre possible au cours de son histoire. L'augmentation des capacités physiologiques et cognitives par la fabrication et l'usage d'artefacts et l'élaboration de savoirs et de savoir-faire n'est donc pas une perspective d'avenir pour l'homme mais bien la définition même de l'humanité. La question se pose seulement de savoir de quelle nature sont ces artefacts, par qui ils sont fabriqués, et dans quelle mesure ils font l'objet d'appropriation par certains groupes aux dépens d'autres groupes.

Les possibilités d'augmentation de soi, et notamment de ses capacités, sont simplement infiniment plus grandes aujourd'hui que par le passé et seront, selon toute probabilité, encore plus grandes demain. Dans le seul domaine médical, on peut dire que les médicaments, les anesthésiques, les divers types de prothèses, les lunettes, les lentilles ou les interventions chirurgicales au niveau de la cornée, 
les greffes de rein, de foie ou de cœur, la neurochirurgie, etc., bref, l'ensemble des dispositifs et techniques médicaux, réparent les organes détériorés, diminuent les douleurs et augmentent notre espérance de vie. Toutes ces avancées ne sont que le produit du développement historique d'une culture de l'artefact, sans comparaison avec celle que l'on trouve déjà présente chez nombre d'espèces animales, et qui commence chez l'homme avec les formes les plus simples de pierres taillées, de lances, de massues, de vêtements, de techniques de production du feu ou d'habitat.

Lorsque Karl Marx parle des luttes pour l' « appropriation privée des moyens de production » - qu'elles concernent les matières brutes et les ressources naturelles ou les outils et les machines -, il ne traite que d'un cas particulier de conquête des positions sociales dominantes en vue d'un meilleur accès à l'ensemble des produits matériels, culturels, techniques, scientifiques, médicaux, administratifs ou bureaucratiques, de la civilisation. Car aux moyens de production s'ajoutent les connaissances pratiques ou théoriques qui permettent de concevoir, de fabriquer et de faire fonctionner les artefacts de toute nature.

Les inégalités, qu'elles soient économiques, résidentielles, alimentaires, vestimentaires, médicales, scolaires ou culturelles, touchent toutes, d'une façon ou d'une autre, à la question fondamentale de l'accès socialement différencié à toutes les extensions de soi possibles, à toutes les formes d'augmentation de sa réalité ou de son pouvoir sur la réalité. Disposer de plus d'espace, de plus de temps, de plus de confort matériel, de plus d'aide humaine, de plus de soins, de plus de connaissances, de plus d'informations sûres, de plus de possibilités de se vêtir, de se reposer ou de se divertir, c'est avoir plus de pouvoir sur le monde et sur autrui.

\section{Conclusion}

Si l'on avait douté de l'importance des inégalités, la pandémie de Covid-19 est venue nous rappeler les probabilités inégales de contamination par le virus (en fonction des conditions de travail et de logement, notamment), les inégalités sociales devant la situation de confinement (selon la taille et la nature des logements, les ressources économiques) ${ }^{2}$ et l'accès inégal, d'un point de vue mondial, à des structures hospitalières, des médicaments et des vaccins. Sur ces derniers points, le Conseil de sécurité de l'ONU a souligné, le 19 mai 2021, la nécessité d'accroître l'aide à l'Afrique face à la pandémie, notamment en matière de vaccins, mais aussi en termes de tests, de médicaments et de traitements adaptés. Il a jugé « préoccupant » le fait que l’Afrique n’ait reçu jusqu'alors que $2 \%$ de tous

2. Concernant ces deux points, on renverra à l'enquête Épidémiologie et conditions de vie (EPICOV) lancée en 2020 auprès d'un large échantillon représentatif de la population française (135000 personnes), par l'INSERM et la DREES (le service statistique du ministère des Solidarités et de la Santé) avec le concours de l'INSEE et de Santé publique France, en vue d'analyser les effets de la pandémie de Covid-19. Elle permet notamment de mesurer les effets des conditions de vie sur l'exposition au virus et, inversement, de saisir les conséquences de l'épidémie en matière de conditions de vie. 
les vaccins administrés à l'échelle mondiale. Et c'est toujours le même Conseil de sécurité qui indiquait que les pays riches, qui ne représentent que $15 \%$ de la population mondiale, avaient profité de $45 \%$ des vaccins produits dans le monde, tandis que l'Afrique n'avait reçu que 1,8\% de ces doses.

Tout cela permet, s'il en était besoin, de prendre conscience des enjeux - qu'on pourrait qualifier, pour une partie d'entre eux, de « vitaux » - attachés à la question des inégalités sociales. Pour celles et ceux qui cumulent tous les pouvoirs et toutes les ressources ou presque, c'est tout simplement le temps de vie qui s'allonge ou le risque de la perdre qui diminue. Les plus grandes conquêtes de l'humanité, qui autorisent à bénéficier d'un confort matériel, des conditions d'hygiène et de tous les soins médicaux et chirurgicaux permettant de prolonger la vie et de diminuer nos souffrances, de continuer à entendre malgré la perte d'audition, de voir malgré la baisse de la vision, etc., sont inégalement accessibles aux pays riches et aux pays pauvres ${ }^{3}$, ainsi qu'aux classes dominantes et aux classes les plus dominées dans toutes les sociétés. 


\section{Références bibliographiques}

Bourdieu P. (1979), La Distinction. Critique sociale du jugement de goût, Paris, Minuit. Chamboredon J.-C. (1996), " La sociologie comme théorie des cultures de classe », Revue européenne des sciences sociales, $\mathrm{n}^{\circ} 103$, p. 116.

Demoule J.-P. (2017), Les dix millénaires oubliés qui ont fait l'histoire. Quand on inventa l'agriculture, la guerre et les chefs, Paris, Fayard.

Hayden B. (2013), Naissance de l'inégalité. L'invention de la hiérarchie, Paris, CNRS.

Lahire B. (dir.) (2019), Enfances de classe. De l'inégalité parmi les enfants, Paris, Seuil.

Marx K. (2007), Le 18 Brumaire de Louis Bonaparte (1852), Paris, Flammarion.

Testart A. (2012), Avant l'histoire. L'Évolution des sociétés, de Lascaux à Carnac, Paris, Gallimard. 